\title{
EAST-ADL - AN ARCHITECTURE DESCRIPTION LANGUAGE
}

Validation and Verification Aspects

Vincent Debruyne ${ }^{1}$, Françoise Simonot-Lion ${ }^{2}$ and Yvon Trinquet ${ }^{3}$

${ }^{I} P S A$ Peugeot-Citroën, 92000 La Garenne Colombe - France; ${ }^{2}$ LORIA (UMR 7503) INPL, Campus Scientifique - BP 239 - 54506 Vandoeuvre-lès-Nancy cedex - France; ${ }^{3}$ IRCCYN (UMR 6597) Ecole Centrale de Nantes, BP 92101 - 44321 - Nantes cedex 3 France.

Abstract: The part of embedded electronic systems in vehicles is nowadays growing. The European EAST-EEA project aims to bring efficient methods and tools for mastering the complexity of these systems. We present EAST-ADL, an Architecture Description Language developed in this project et show how the verification and validation activities are linked to this language.

Keywords: ADL, Verification, Validation, Requirements, Real-Time Systems.

\section{INTRODUCTION}

For economical and technological reasons, the part of electronic and software is increasing significantly in automotive systems. The main characteristics of these systems are their distributed nature and the fact that they have to provide a level of quality of service fixed by the market, the safety requirements and the cost requirements. Furthermore, their development process is shared between different partners. Therefore their development and their production have to be based on a suitable methodology including modelling, validation, optimisation and test. Obviously, any error detected during the integration step leads to a costly feedback on the specification or design activities, and it must be avoided. So, in order to improve the quality of the design process, new methodologies are emerging. In particular, the actors implicated in the development of a system apply more and more methods and techniques ensuring the correctness of 
subsystems as early as possible in the design stages and a new trend is to consider the integration of subsystems at a virtual level ${ }^{1}$. This means that each partner involved in the development process will be able to design, prove and validate the models of each subsystem with respects to the requirements. Then the challenge is the validation of the whole system in a cooperative way. This paper demonstrates how the EAST-EEA project ${ }^{2}$ brings some solutions to this challenge.

Specifically, the section 2 describes some approach related to this problem. A brief presentation of the context, the objectives and main results of EAST-EEA project is given in section 3. In section 4 we present how the validation and verification activities can be modeled in a consistent way all along the development process while section 5 focuses on performance properties and specifies how the model of an embedded system has to be completed in order to allow the verification process of this kind of properties. Section 6 brings some conclusions on this proposal.

\section{RELATED WORKS}

The way to improve the quality and the flexibility of an embedded electronic system while decreasing the development and production cost is to design and validate it at a virtual level. The development of complex systems requires frameworks that support functional and extra-functional specification at different steps of the development and methods for ensuring the system correctness. Therefore, the problem is, on the one hand, to identify the abstraction level at which the components and the whole system should be described. And on the other hand, in order to ensure the system correctness, we have to identify the validation / verification activities or the automatic generation techniques to apply. Consequently, we have to identify the formalisms supporting the identified models. Some main keywords are related to this problem: 1- architectures, referring to the Architecture Description Language concept (ADL), well known in computer science; 2components, leading to modularity principles and object approach and 3Model Driven Architecture ${ }^{3}$, well-suited to generation of correct implementations.

An Architecture Description Language is an approach for software and system architecture specification ${ }^{4}$. In the avionic context, MetaH ${ }^{5}$ developed at Honeywell, has been chosen, in 2001, as the basis for the definition of an Avionics Architecture Description Language (AADL) standard under the SAE authority ${ }^{6}$. The core AADL supports system modeling and execution platforms. It provides a way for describing control, data flow, some non functional aspects (timing requirements, fault and error behaviors, 
time and space partitioning, safety and certification properties). Efficient tools are provided with AADL for verification purpose as schedulability analysis.

The Cotre Project ${ }^{7}$ targets the same objective. It provides a methodology for the development of real-time avionic systems by specifying in a formal way the links between the description of such a system expressed thanks to an Architecture Description Language and the formal techniques relying to verification activities. The formalisms used for verification activities are Transition Systems, Time Automata and Time Petri Nets (tools Uppaal, Tina or Aldebaran).

In automotive industry, recent efforts brought a solution for mastering the design, modeling and validation of embedded systems. A first result was obtained by the French project AEE (Embedded Electronic Architecture) ${ }^{8}$. A result of this project is a language named AIL_Transport (Architecture Implementation Language for Transport). It allows the specification in the same framework of embedded architectures at several abstraction levels. The highest one captures the requirements and give a functional view. The lowest level models an implementation ${ }^{9},{ }^{10}$. Two tools were developed in order to automate scaling and verification activities. They take, as input data, the system description in AIL_Transport. The first one is devoted to optimal distributed code generation realized by Syndex tool ${ }^{11}$. The second one is dedicated to performance property verification by using Opnet simulator (www.opnet.com.). A similar work is proposed in CAROSSE ${ }^{12}$ through a language for implementation description (tasks exchanging messages over a communication architecture) and a timing property verification tool hiding the complexity of the required models.

The CLARA ${ }^{13} \mathrm{ADL}$ is a general purpose language developed for the design of asynchronous reactive real-time systems. Special attention was paid to the control flows. Indeed CLARA allows to express complex synchronization and activation laws, and timing requirements. Tools based on Time Petri Nets can be used for verification activity at the operational level.

Face with these works, the ITEA European project EAST-EEA, (July 2001 - June 2004), involving carmakers, suppliers and research institutes, investigates automotive embedded architectures and development aspects. It involves and aims to unify the concepts for automotive software development and moves towards a common notation, the whole approach being supported by suitable verification and validation (V\&V) tools. Among the addressed, we focus in the next sections on the Architecture Description Language, named EAST-ADL, that was specified and on its use for the validation and verification activities. GME2000 tool $^{14}$ supports the meta model describing EAST-ADL. 


\section{EAST-ADL}

The purpose of EAST-ADL is to provide a support for the nonambiguous description of in-car embedded electronic systems at each level of their development. It provides a framework for the modeling of such systems through 7 views as shown in Figure $1^{15}$. Each view, except the first one, capture an "architecture" this term being used with the sense of an entity organization. Each view is the result of a specific analysis among the development process: in EAST-ADL terminology it is called an artifact.

\subsection{Abstraction layers supported by EAST-ADL}

The EAST-ADL abstraction layers are quickly described hereafter.

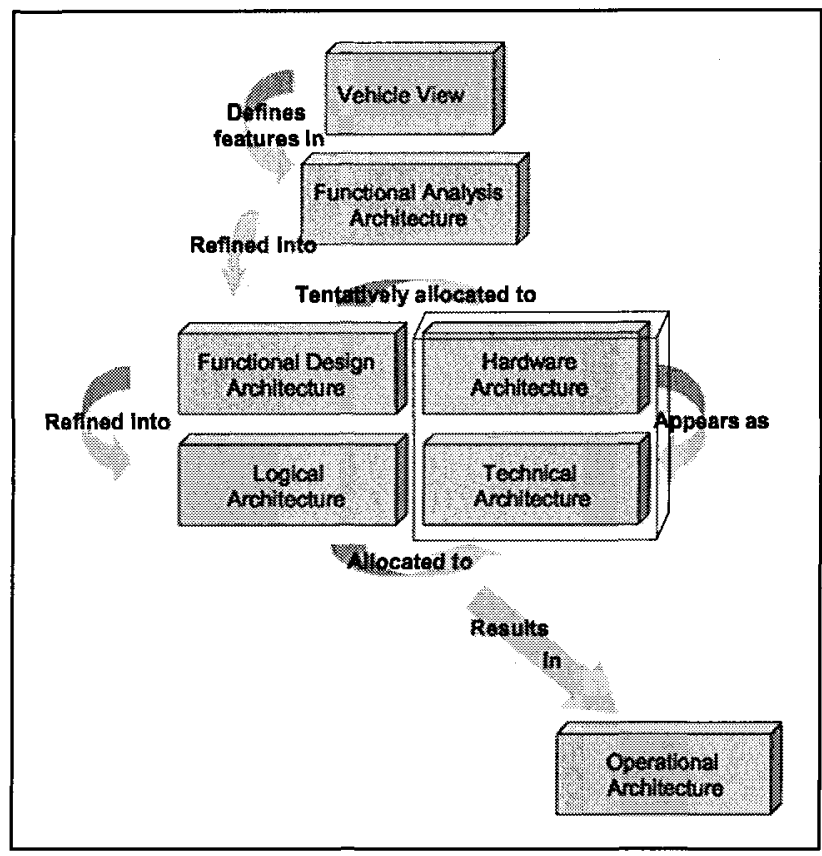

Figure 1. The abstraction layers of the EAST ADL.

- Vehicle View is the level where user visible features are described. Examples of such features are anti-lock braking or windscreen wipers;

- Functional Analysis Architecture level represents the functions realizing the features, their behavior and their cooperation. There is an n-to-n mapping between Vehicle View entities and Functional Analysis Architecture entities, i.e. one or several functions may realize one or several features; 
- Functional Design Architecture level models a decomposition or refinement of functions described at Functional Analysis Architecture level in order to meet constraints regarding allocation, efficiency, re-use, supplier concerns, etc. Again, there is an n-to-n mapping between entities at Functional Design Architecture level and the corresponding ones at Functional Analysis Architecture level;

- Logical Architecture is a flat software structure where the Functional Design Architecture entities have been instantiated. This level provides an abstraction of the software components to implement on the Technical Architecture. The logical architecture contains the leaf functions of the Functional Design Architecture. From the Logical Architecture point of view, the code could be automatically generated in many cases.

In order to model the implementation of a system, EAST-ADL furnishes, on the one hand, a way for the description of the hardware platforms and their available services (operating system, protocols, middleware) and, on the other hand, a support for the specification of how a logical architecture is distributed onto a platform. For this purpose, three additional views are necessary:

- The Hardware Architecture level includes the description of the ECUs (Electronic Component Unit) and more precisely those of the used microcontroller, sensors and actuators, the communication links (serial links, networks) and their connections.

- At Technical Architecture level the model of the operating system and/or Middleware API and the services provided (schedulers, frame packing, memory management, I/O drivers, diagnosis software, download software etc.) are given. So programmer's view of the Hardware Architecture is given by the Technical Architecture.

- The Operational Architecture models the tasks, managed by the operating systems and frames, managed by the protocols. It is the result of the mapping of the Logical Architecture entities onto the Technical Architecture. At this lowest abstraction level, all implementation details are captured.

A system described at the Functional Analysis level may be loosely coupled to hardware. Indeed it may be based on intuition, various known constraints or as a back annotation from more detailed analysis on lower levels. Furthermore, the structure of the Functional Design architecture and of the Logical Architecture is aware of the Technical architecture. Finally, this EAST-ADL provides the consistency within and between artifacts belonging to the different levels, at a syntactic and semantic point of view. 
This leads to make an EAST-ADL based model a strong and non-ambiguous support for building automatically models suited to formal validation and verification activities.

The compliance to UML2 was a constraint for the definition of the EAST-ADL language. A prototype, based on GME2000 tool ${ }^{14}$, was realized. It provides, at present, an editor for each view previously described and a checker verifying the consistency of a model, according to EASTADL semantic. A complete model of a system is stored in an XML database.

The description of the language elements was divided into six parts corresponding to different language domains, as "structure domain" for the language elements describing the structural relation, or "behavior domain" for the language elements describing the behavioral models. In the next parts of the paper we give some information on the "requirement domain" and the "V\&V domain".

\subsection{Requirements modeling}

As EAST-ADL supports all the activities done along the development process of an automotive embedded system, it provides a way for expressing the requirements that guide the building of a solution at each step of this development. So EAST-ADL defines the different requirements types that can be used. It allows to link them on the one hand to components defined at one or several architectural views and on the other hand to analysis models, design models and implementation models ${ }^{16}$. Finally the tracing activities between different requirements or between different versions of requirements can be expressed in this language.

Five types of requirements were identified. Each of them is characterized by a textual description possibly completed by a formal description, information supporting the tracing activities and a status which is to be set to specific values according to the result of particular design, validation or verification activities. The requirement types are:

- EFeatures: an EFeature object (EFeature) describes the required functionalities of an embedded system; this kind of object is used, mainly, for specifying the system at Vehicle and Functional Analysis Architecture levels. EFeatures may be decomposed into sub-features or variant features ("variant" stands here for the various type of equipments for a car);

- Interactions: this type is used to specify the cooperation modes between EFeatures through textual description, semi formal one (as use-cases) and possibly formal one (for example, Message Sequence Charts);

- Functional Requirements: this type aims to specify the behavior of EFeatures by means of a set of required properties; once more, a formal 
description (state-transition diagrams, Messages Sequence Charts, ...) can complete the textual one;

- Design Constraints: this is a kind of requirement that constraint the research field for a solution; for example, such a constraints can impose a communication standard, a legacy tool for designing the system or a criteria to optimize (cost, power consumption, network bandwidth, ...);

- Quality Requirements: they are used to express extra-functional properties; among these Quality Requirements, we can cite performance properties, reliability properties, safety properties, ...

In this paper, we will focus on the Quality Requirements and on their links to formal validation and verification activities. For this purpose, we recall, in the following section, the main classes of validation and verification techniques that are used in automotive industry and how the validation and verification process can be modeled.

4. VALIDATION AND VERIFICATION ACTIVITIES

\subsection{Main Validation and Verification techniques used in automotive industry}

From an industrial point of view, two main objectives for validation and verification can be identified:

- Validation and verification of all or parts of a system at a functional level without taking into account the implementation characteristics. These activities ensure the consistency of the system mainly with respect to the EFeatures, Interactions and Functional Requirements. At this level, simulation or formal analysis techniques can be used;

- Verification of properties of all or part of a system at operational level. These activities take into account the performances of both the hardware and Technical Architectures and also the load that is due to a particular allocation of the Logical Architecture on the Technical Architecture. This objective can also be reached through simulation and formal analysis techniques. Note that, in this context the formal approach for the verification of the feasibility of a set of tasks and frames is done through a timing analysis method.

For these purposes, a model suited to the concerned techniques and to the associated tool has to be built. The way used to generate such a model will 
be explained in section 5. Some tools are of course of general interest in this context as, for example Matlab / Simulink or Stateflow as well as Statemate. In some cases, an interface encapsulates these tools in order to adapt the tool to the automotive context.

Moreover, these techniques that work on virtual platforms are completed by test techniques in order to verify that a realisation is correct. We can cite the test of software components, the test of logical architectures and the test of an implemented embedded system. Note that the testing activities as well as the simulation ones consist in providing a scenario of events and/or data that stimulate the system under test or stimulate an executable model of the system; then, in both techniques we have to look which events and/or data are produced by the system. The input scenario can be manually built or formally generated. In this last case the test or simulation activity is closely linked to a formal analysis technique.

\subsection{Validation and Verification Process}

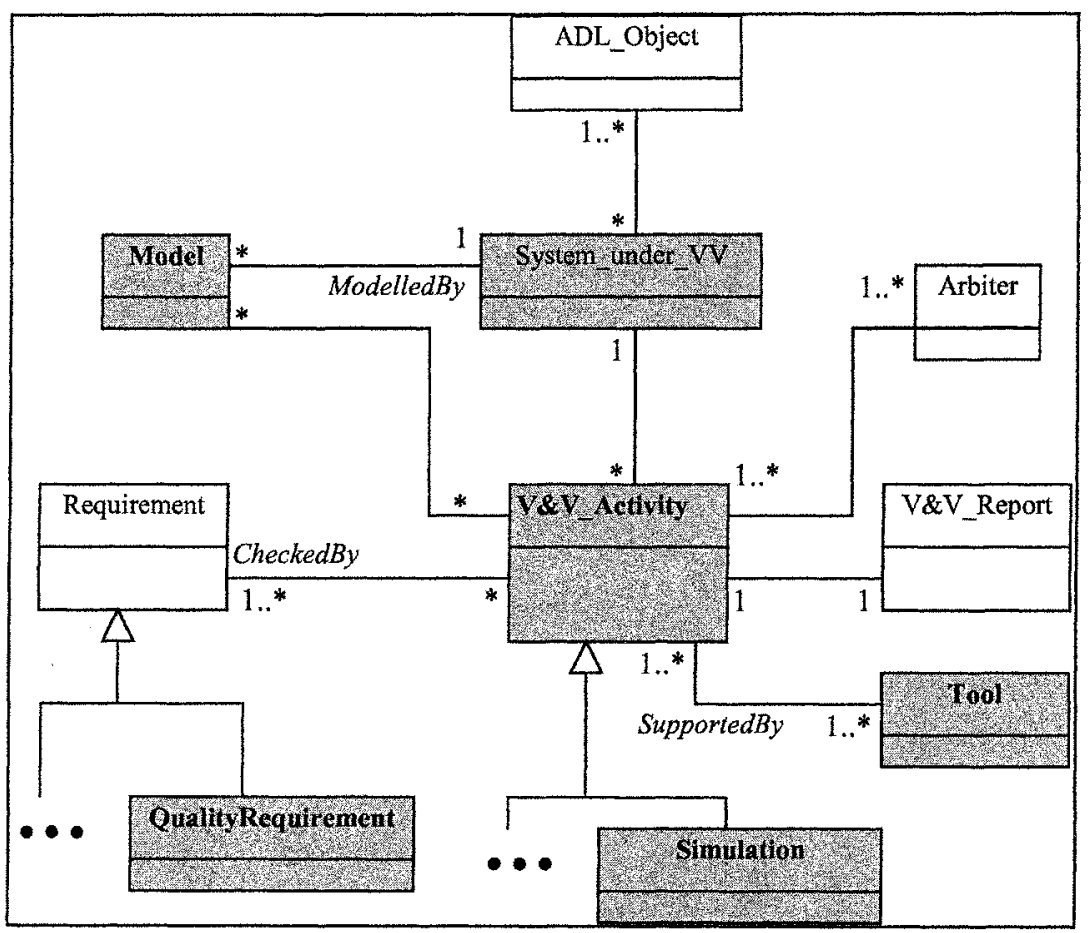

Figure 2. Validation and Verification process model 
The EAST ADL language provides a way for supporting the Validation and Verification activities along the development process.

A main purpose is to formalize the relations between $V \& V$ activities and the other items supported by EAST ADL. These relations are illustrated by the figure 2 . This description is largely inspired by the testing profile defined in UML2. In this figure, the classes that will be used in the case study are highlighted (V\&V_Activity, System_under_VV, Model, Tool, QualityRequirement).

In particular, two links should be outlined:

- a first one with Requirement objects: the specified association establishes that one V\&V activity can contribute to the checking of several requirements, while one requirement can be checked thanks to several V\&V activities;

- a second one with embedded architecture objects: obviously, as the purpose is to validate or verify properties of all or part of an embedded system, a $V \& V \_$Activity object is associated to a set of objects related to an architecture layer. For example, a timing analysis applied to one Electronic Control Unit (one micro-controller) is concerned by the set of tasks (objects TaskOS) that are local to this ECU and by the scheduling policy used on this ECU and described by an OperatingSystem object. This set of ADL objects is named System_under_VV.

Furthermore, as introduced in the previous section, a V\&V activity is a generic class that can be refined in several subclasses, modelling formal analysis or scenario-based techniques (simulation or test).

Because the way to conduct V\&V activity depends on the techniques, several entities and associated attributes have been identified. Formal analysis is mainly based on a formalism, a property to verify and a verdict concerning the verification of the property. Test (or simulation) is based on a scenario, acceptable results corresponding to this scenario and a verdict which is elaborated thanks to actual results of the test (or simulation). $V V_{-}$Report and Arbiter model the final verdict of a $\mathrm{V} \& \mathrm{~V}$ activity.

\section{CASE STUDY: PERFORMANCE PROPERTY VERIFICATION}

EAST-ADL supports the consistency between architectural objects, requirements and validation or verification activities. We illustrate this by studying how can be applied the schedulability analysis of frames over CAN 
under error pattern assumption. In fact, we show two main characteristics of EAST-ADL. On the one hand, we identify how EAST-ADL supports the consistency between requirements specified at different steps of the development process and the verification activities used to ensure that these requirements are checked. On the other hand, as for any verification activity a model of all or part of the system has to be built, we demonstrate how this building process can be automated by exploiting the semantic of objects and interactions expressed in EAST-ADL.

Let us consider, as an example, two quality requirements specified during the development of an embedded system.

- QR1: "the system has to be tolerant to EMI perturbation following a profile due to a given type of radar"; this requirement can be given in the earliest steps of the development process.

- QR2: "a freshness constraint (2 milliseconds) is imposed to the signal $<$ VehicleSpeed $>$; the average of respected freshness constraint has to be more than $80 \%$ "; this is required at functional level; let us assume that this signal $<$ VehicleSpeed $>$ is produced by a function and consumed by another one, so this requirement can be translated in "the percentage of missed deadlines $(2 \mathrm{~ms}$.) for the exchange of signal $<$ VehicleSpeed $>$ has to be less than $20 \%$ ".

These two requirements impose to the designer the verification of just one property at operational architecture level: the probability that the frame containing the signal $<$ VehicleSpeed $>$ misses its deadline $(2 \mathrm{~ms}$.) has to be less than $20 \%$ (we suppose here that producer and consumer of $<$ VehicleSpeed $>$ signal are deployed on two different nodes).

A way to check these two quality requirements for a system is to apply an analytical method for performance evaluation of the network that supports the exchange of the concerned signal as illustrated in figure 4. A tool, named VACANS, is available for this performance evaluation. It is based on a recurrent algorithm whose principle was given first by Tindell et al. ${ }^{17}$ and that was extended for taking into account a more realistic error model by Navet ${ }^{18}$ as needed in this verification requirement. It takes as entry, on the one hand, some characteristics of the CAN network and the specification of the frames that it supports and, on the other hand, some parameters for modelling the error occurrences due to a given EMI perturbation pattern. VACANS evaluates the worst-case deadline failure probability for each frame. The term worst-case is justified by two assumptions: each error is detected on the last bit of the frame and the time needed by each frame to 
gain the bus is the maximum possible. More precisions about this technique can be found in ${ }^{18}$.

So, for checking the requirements $\mathrm{QR} 1$ and $\mathrm{QR} 2$, a verification activity named EMI_Tolerance, can be done by running VACANS tool. The result of this activity (worst case deadline failure probability for each frame) can then be analysed and a report that establishes the verdict about the property is produced. Note that several other results can be obtained as for example, the percentage of missed deadlines for each other frame, the mean bandwidth of the network, ... So, we consider in EAST-ADL that all these results contribute to a general evaluation report which can be linked to several verification activities, in particular, in this example, to EMI_Tolerance object.

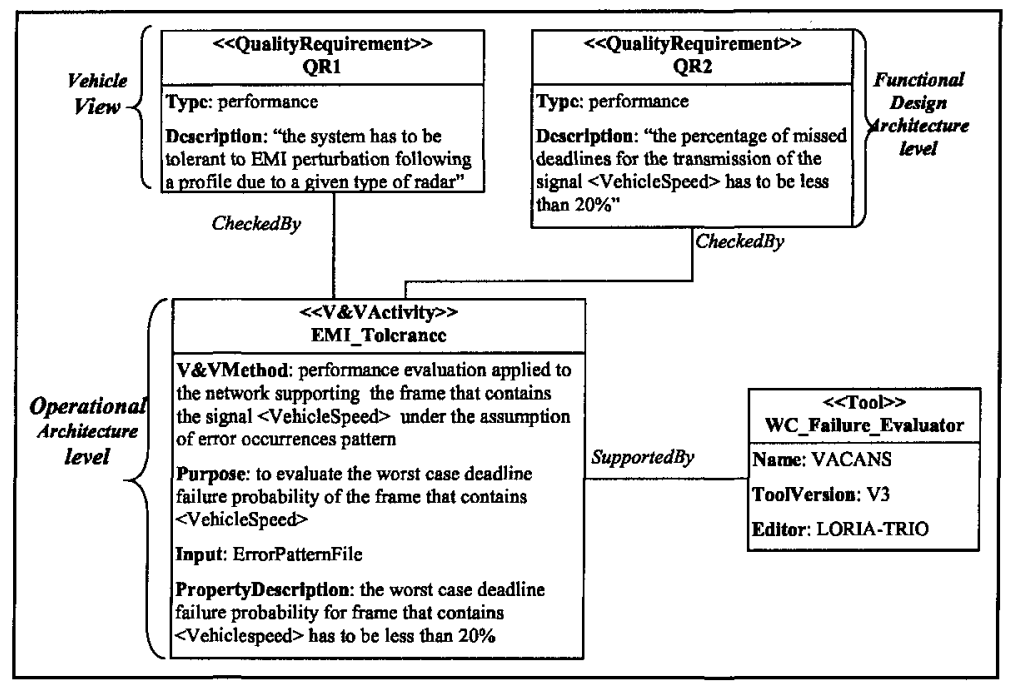

Figure 3. How quality requirements QR1 and QR2 are checked through a V\&V activity

As shown previously, EMI_Tolerance realised thanks to VACANS tool, is applied to a set of related objects described in EAST-ADL. We illustrate in the following how these objects can be "extracted" from the EAST-ADL compliant repository. 


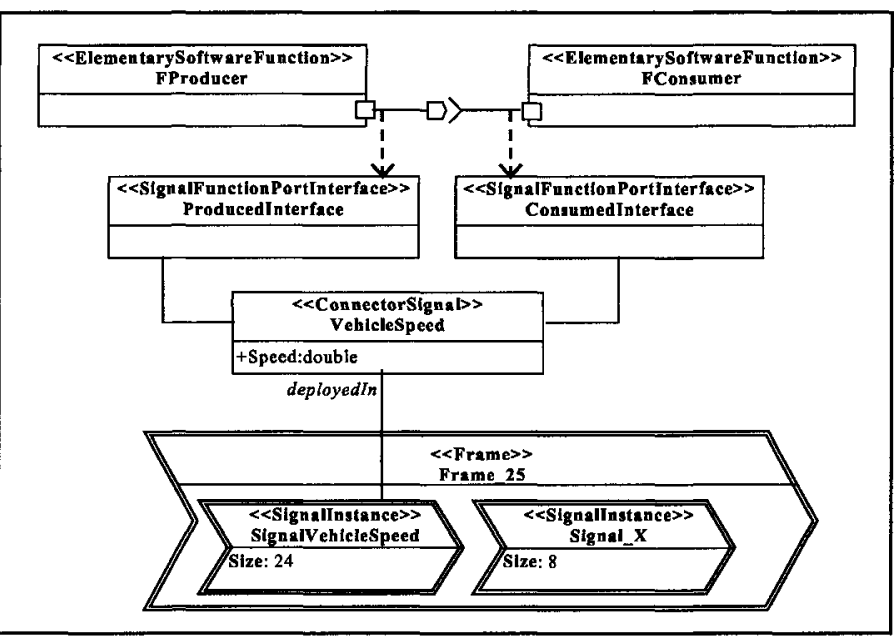

Figure 4. From the VehicleSpeed to the object Frame that contains its realization

From functional level (a signal) to operational level (a frame).

The entry point for this extraction is the object ConnectorSignal whose name is <VehicleSpeed $>$. This signal, its producer and consumers were identified at a functional level (see figure 4). In a following step, producer $(<F$ Producer $>$ ) and consumer $(<F$ Consumer $>$ ) of the signal were deployed in OSTasks allocated to different ECUs (Electronic Control Unit). Therefore, the ConnectorSignal <VehicleSpeed $>$ is transformed in a SignalInstance object, named <SignalVehicleSpeed $>$, whose size is 24 bits and is deployed in an object Frame named $\langle$ Frame_25 $\rangle$. The object under verification is this frame.

From one frame at operational level to the network supporting this frame.

The VACANS tool obliges us to gather all the frames that share the same network. For this purpose, from the object Frame_25, we obtain the object network supporting this frame.

\section{From a network to all the frames sharing it.}

Then, this object, named here CAN_Chassis, allow us to obtain the set of frames that we have to take into account for the verification activity (see figure 5).

Each Frame in EAST-ADL is characterised by several global attributes:

- Period: the trigger period (in $\mathrm{ms}$.); it represents the nominal period for time triggered frames and the minimum inter-arrival time for event triggered ones; 
- Offset: (in ms.); it gives the offset from the starting time; this attribute is only applied for time triggered frames;

- FrameSize: (in bits) the total length of the frame;

- DataSize: (in bits) the sum of the size of each object SignalInstance deployed in this frame.

These attributes can be extracted automatically from the repository describing the embedded system in EAST-ADL. Nevertheless, some other characteristics have to be completed before using VACANS. In particular, due to the object CAN_Chassis - whose protocol is CAN - we have to complete the characteristics of each frame by the attribute Priority. Furthermore, for establishing the verdict, a deadline characteristic is added to the Frame_25 (2ms. as explicitly given in the requirement QR2); as nothing is given for the other frames, we consider that their deadlines are equal to their periods.

The object CAN_Chassis has to be completed by several attributes:

- Throughput: in bits/s;

- Length: in $\mathrm{m}$.

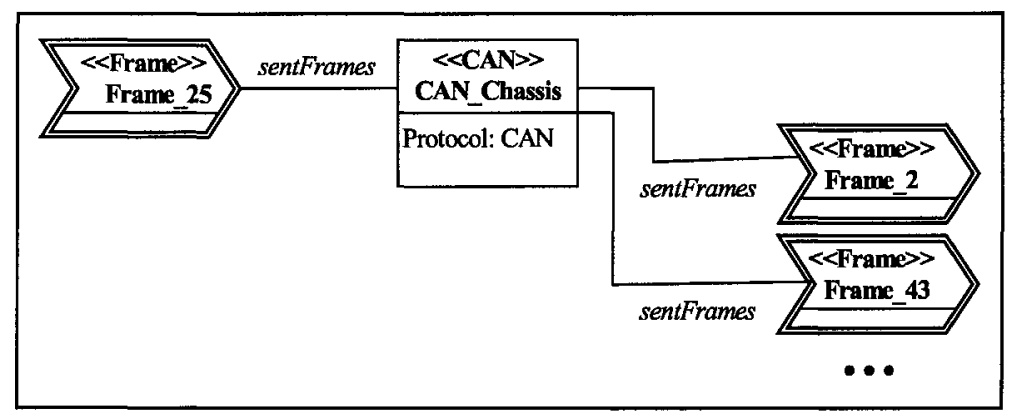

Figure 5. From Frame_25 to all the frames sharing the same network

Finally, the use of Vacans requires to fix the parameters used for specifying the errors occurrences according to a generalized Poisson Process ${ }^{18}$. These parameters are $\langle\lambda, u, \alpha\rangle$ where the inter-arrival between two perturbations is given by $\exp (\lambda)$; the length of a burst is given by the number of errors $u$ and, when an error occurs, $\alpha$ is the probability that it is a burst of errors and $1-\alpha$ is the probability that it is a single error (these parameters are illustrated in figure 6). The algorithm computes then the worst-case deadline failure probability for each collected frames. 


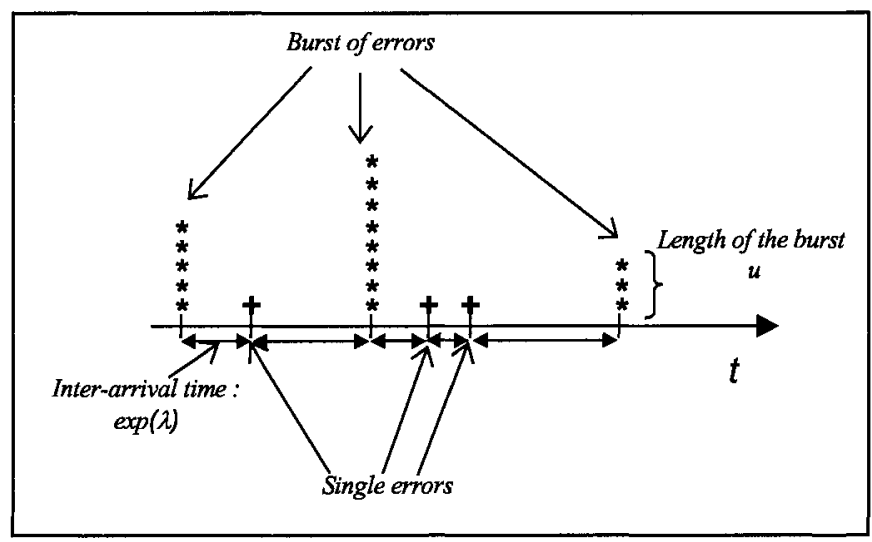

Figure 6. Parameters of error distribution

The complexity of the automatic generation of formal model used, in this case, as an entry of VACANS tool depends on the structure of the XML database. In the present prototype, the organization of the XML database is such that it needs for this case study:

- to browse a first time the XML file in order to find the object VehicleSpeed (ConnectorSignal object); the object SignalVehicleSpeed (SignalInstance object) is directly referenced;

- to browse a second time the XML file in order to find, among the objects Frame, the one that is connected to SignalVehicleSpeed; the object CAN_Chassis (CAN object) is directly referenced;

- to browse a third time the XML file, among the objects Frame, in order to find all the objects that are connected to CAN_Chassis object.

This example demonstrates that thanks to the semantic of the objects described according to EAST-ADL, some activities can be automated and that formal links connect objects at different levels. So EAST-ADL is not only a support for the non-ambiguous modeling of an electronic embedded system but also for its development process.

\section{CONCLUSIONS}

In this paper we have presented a few concepts of the EAST ADL common modeling language for automotive software development. Currently a major version of the language has been defined and used in demonstrators in the course of EAST-EEA project. This will probably give 
good expertise for language evolutions. The paper has focused on requirements and $V \& V$ aspects through an example at the operational architecture level. This example shows how some V\&V activities can be automated and that formal links connect objects at different levels. Ongoing works are on the specification of the needed language attributes for various V\&V methods as timing analysis, or model-checking.

\section{REFERENCES}

1 P. Giusto, J.-Y. Brunel, A. Ferrari, E. Fourgeau, L. Lavagno and A. SangiovanniVincentelli, Automotive Virtual Integration Platforms: Why's, What's, and How's, Proceedings of the International Conference of Comp. Desc. , July 2002.

2 ITEA - EAST EEA Project, www.east-eea.net/docs.

3 Object Management Group, Model Driven Architecture, http://www.omg.org/mda/

4 R.N. Taylor, N. Medvidovic, A Framework for Classifying and Comparing Architecture Description Languages, Tech. Report, University of California, Irvine, 1997.

5 S. Vestal, MetaH Reference Manual, Tech. Report, Honeywell Technology Center, 1995.

6 P. H. Feiler, B. Lewis, S. Vestal, The SAE Avionics Architecture Description Language (AADL) Standard, RTAS 2003, Washington, 2003.

7 B. Berthomieu, PO. Ribet,F. Vernadat, JL. Bernatt, JM. Farines, JP. Bodeveix, M. Filali, G. Padiou,P.Miche, P. Farail, P. Gaufillet, P. Dissaux, J.-L. Lambert, Towards the verification of RT systems in avionics: the Cotre approach, El. Notes in Th. Comp. Science 80, 2003.

8. AEE, Architecture Electronique Embarquée, http://aee.inria.fr, 1999.

9. J.P. Elloy, F. Simonot-Lion, An Architecture Description Language for In-Vehicle Embedded System development, 15th IFAC World Congress, Barcelona, Spain, 2002.

10.J. Migge, J.P. Elloy, Embedded electronic architecture, Proceedings of 3rd International ,Workshop on Open Systems in Automotive Networks, Bad Homburg, Germany, 2000.

11 R.Kocik, Y. Sorel, A methodology to design and prototype optimized embedded robotic systems, 2nd IMACS International Multiconference CESA'98, Hammamet, Tunisia, 1998

12 P. Castelpietra, YQ. Song, F. Simonot-Lion, Analysis and simulation methods forevaluation of a networked embedded architecture, IEEE Trans. on Industrial Electronics, 49-6, 2002.

13 Durand E., Description et vérification d'architectures d'application temps réel : CLARA et les réseaux de Petri Temporels. PhD thesis, Ecole Cdentrale de Nantes, 1998 (report in French).

14 A. Ledeczi, M. Maroti, A. Bakay, G. Nordstrom, J. Garrett, C. Thomason, J. Sprinkle, P. Volgyesi, GME 2000 Users Manual (v2.0), technical report, Vanderbilt University, 2001.

15 U. Freund, O. Gurrieri, J. Küster, H. Lönn, J. Migge, M.-O. Reiser, T. Wierczoch and M. Weber, An Architecture Description Language for Developing Automotive ECUSoftware, INCOSE 2004, International Conference On Systems Engineering, Toulouse, France, june 2004.

16 M.Weber, J.Weisbrod, Requirements Engineering in Automotive Development Experiences and Challenges. IEEE Software, vol. 20, no. 1, pp 16-24, 2003.

$17 \mathrm{~K}$. Tindell and A. Burns, Guaranteeeing message latencies on Controller Area network (CAN), $1^{\text {st }}$ International CAN Conference, ICC'94.

18 N. Navet, Y.-Q. Song, F. Simonot, Worst-case deadline failure probability in real time applications distributed over CAN, in Journal of System Architecture, 46(7), 607-618. 